\title{
Disinhibition and external inhibition in fixed interval operant conditioning'
}

\author{
Bruce Flanagan, UNIVERSITY OF SOUTH FLOPIDA \\ Wilse B. Webb, UNIVERSITY OF FLORIDA
}

\begin{abstract}
Probe stimuli were introduced at $10 \mathrm{sec}$. and at 40 sec. in a one minute fixed interval operant conditioning paradigm. Data indicated disinhibition resulting from the 10 sec. probe. Some evidence for external inhibition was seen as a result of the $40 \mathrm{sec}$. probe.
\end{abstract}

\section{Introduction}

Kimble (1961) in his discussion of the similarities between classical and instrumental conditioning suggests that there may be a parallel between long trace and/or long delay classical conditioning and the fixed interval instrumental paradigm in regard to "inhibition of delay." He notes that "whether this is more than a superficial similarity depends upon whether the two processes respond in the same way to the same experimental operations', (p. 65). He then describes the disinhibition which occurs in the classical conditioning paradigm as the result of an additional stimulus at the beginning of the delay interval, i. e., the reappearance of the CR early in the interval which the $\mathrm{S}$ has, as a result of conditioning, learned to delay (inhibit) until the later part of the interval. Kimble then concludes: "Tests for similar effects during responding on a fixed interval schedule have not been made. This lack suggests an important area for future experimentation', (p. 65).

This paper reports the results of the introduction of a "probe" or extra stimulus in the fixed interval period.

\section{Subjects}

The Ss were five experimentally naive female rats of the Wistar strain, from the University of South Florida colony. All Ss were 115-120 days old at the beginning of pretraining. They were housed individually and maintained on ad lib dry Purino chow. Ss were water deprived for $23 \mathrm{hr}$. before sessions.

\section{Apparatus}

The experimental chamber was $14 \times 10 \times 10$ in, built from aluminum sheet metal with a stainless steel grid floor. A 2 in response bar projected from the rear wall 3 in above the grid, and required $40 \mathrm{gm}$ of downward pressure to activate. The reinforcement device, a water dipper presented $0.1 \mathrm{cc}$ of water for $3 \mathrm{sec}$. This was positioned in the floor 3 in to the left of the response bar. A 15-w light contained in the ceiling was turned on when the dipper was presented and off when withdrawn. White noise $85 \mathrm{db}$ (re: SPL) was constant throughout all conditions.

A system of relays and timers automatically programmed the experimental procedures; responses and reinforcements were recorded on a cumulative recorder and on electrical impulse counters.

Two kinds of probe stimuli, noise and vibratory, were used. The noise stimulus was a $1000 \mathrm{cps}$ square wave presented at $90 \mathrm{db}$ (re: SPL). The vibratory stimulus was produced by a small solenoid fixed to the outside of the experimental chamber, vibrating at $2 \mathrm{cps}$. This stimulus was empirically judged to "just produce a looking response" with 3 naive rats.

\section{Procedure}

Pretraining: Dipper training was carried on for three 12-hr. sessions with the reinforcement programmed randomly, using a mean value of $5 \mathrm{~min}$. between presentations. The rats were then shaped through the method of successive approximation to press the response bar for water. Continuous reinforcement followed for ten $1-\mathrm{hr}$. sessions. The animals were then switched to a 1-min. fixed interval reinforcement schedule and run for $151-\mathrm{hr}$. sessions. This was done to obtain stable fixed interval performance. The noise probe was introduced on the 16 th FI session.

\section{Noise Probe}

In these sessions probes of two durations were used: $\mathrm{A}$, onset $10 \mathrm{sec}$. after previous reinforcement; and $\mathrm{B}$, onset $40 \mathrm{sec}$. after previous reinforcement. Both probes remained on until the next reinforcement was obtained. The sequence was as follows: Pre-Base, 30 reinforcements/Probe A/Inter-Base, 12 reinforcements/Probe $\mathrm{B} /$ Inter-Base, 10 reinforcements/Probe B/Inter-Base, 8 reinforcements/Probe A/Inter-Base, 11 reinforcements/Probe B/Inter-Base, 9 reinforcements/Probe A/Post-Base, 10 reinforcements.

\section{Vibratory Probe}

Following a four-week time lapse, Ss were run using the vibratory probe. The probe sequence was identical to that used with noise. S-5 was not run this session because of apparent signs of respiratory sickness.

\section{Results}

The results are shown in Fig. 1 and 2. These figures present the mean number of responses occurring in the presence of the noise probe and in the presence of the vibratory probe. The Pre-Base response means are the means of the responses occurring in each $10-\mathrm{sec}$. interval for the 30 reinforcement intervals prior to the first probe. There were, then, 150 response periods in each interval for the noise probe group and 120 response periods in each interval in the vibratory probe group. The Post-Base response means are based on the 9 reinforcement intervals following the trial after the last probe (45 and 36 response periods respectively). The means of the A probe and B probe are based upon the 
responses occurring during the 10-sec. intervals within the 1-min. intervals during which the 3 probes of an $A$ type (10 sec. after the interval hadbegun) or the 3 of the B type (40 sec. after the interval had begun) had been introduced.

\section{Discussion}

Both the noise and the vibratory probe, when introduced at the 10-sec. point, may be interpreted as producing disinhibition of the inhibition of delay represented in the Pre- and Post-Base periods. Furthermore, in the case of the vibratory probe and suggested in the case of the noise probe, there were results similar to the Pavlov study (cited by Kimble) in which the effect of the additional CS "was to disinhibit the response during the first

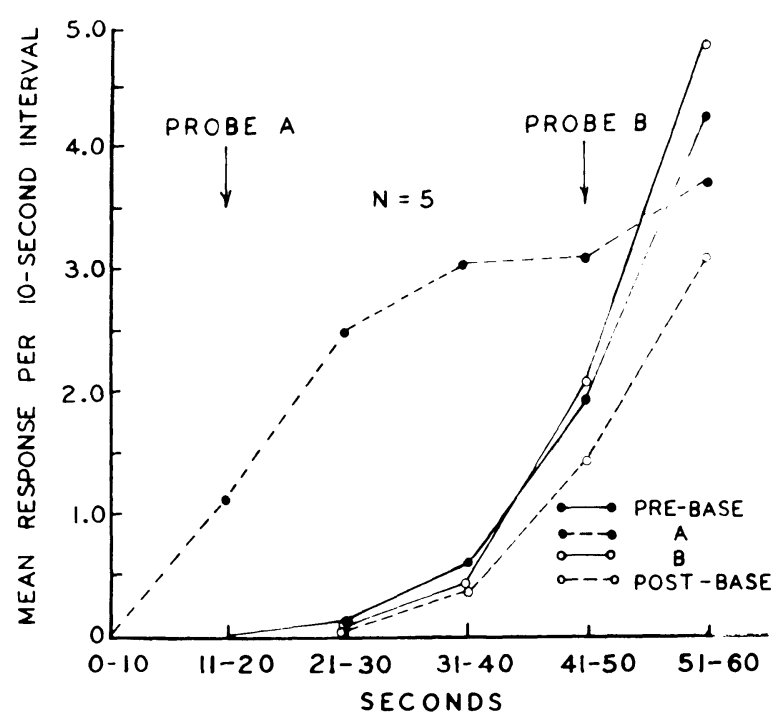

Fig. 1. Noise probe.

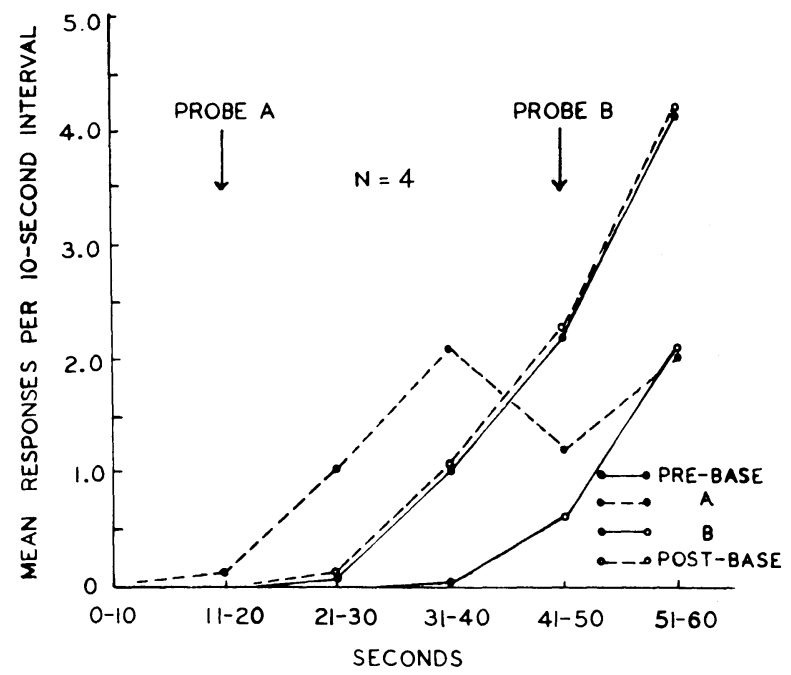

Fig. 2. Vibratory probe.

$1.5 \mathrm{~min}$. on this trial and externally inhibit the CR for the last 1.5 min."

As with the 10-sec. probe, the 40-sec. vibratory probe (which we hypothesized would result in external inhibition) yielded supporting evidence for the appearance of external inhibition. Unfortunately, these results did not occur in the case of the noise probe, a discrepancy for which we are unable to account.

\section{References}

KIMBLE, G. Hilgard and Marquis' conditioning and learning. New York: Appleton-Century-Crofts, 1961.

\section{Note}

1. We express our appreciation to Henry Pennypacker for his comments and criticism. 